\title{
Usability und Smart Home: Aktuelle Herausforderungen und Implikationen
}

\author{
Sandra Schering \\ itemis AG \\ Am Brambusch 15 \\ 44536 Lünen \\ sandra.schering@itemis.de \\ Michael Jendryschik \\ itemis AG \\ Am Brambusch 15 \\ 44536 Lünen \\ michael.jendryschik@itemis.de
}

\author{
Jasmin Kuhn \\ itemis AG \\ Am Brambusch 15 \\ 44536 Lünen \\ jasmin.kuhn@itemis.de
}

\begin{abstract}
Smart Home wird immer mehr zu einem Mainstream-Thema. Während sich früher vor allem technikaffine Personen mit dem Thema auseinandergesetzt haben, wird heute auch in „,normalen“ Haushalten für den Einsatz entsprechender Hard- und Softwarelösungen geworben. Dies impliziert als nur eine von vielen Herausforderungen, dass die Lösungen einer breiten Nutzergruppe mit verschiedenen Eigenschaften zugänglich sein müssen. Im Rahmen des Usability-Engineering-Prozesses einer SmartHome-Anwendung haben wir durch verschiedene Evaluationen solche Herausforderungen und Fragestellungen identifiziert und diskutieren in diesem Paper mögliche Lösungsansätze.
\end{abstract}

\section{Keywords}

Usability, Smart Home, Internet of Things

\section{Einleitung}

Smart-Home-Lösungen durchdringen immer mehr den Massenmarkt. Während laut statista (2015) im Jahr 2014 3,3 Millionen Smart-Home- und Hausautomatisierungslösungen in Europa installiert wurden, werden für 2017 17,4 Millionen und für 2019 bereits 29,7 Millionen Installationen erwartet. Auch im Alltag wird durch Plakat- und Fernsehwerbung immer mehr für das Thema, dessen Möglichkeiten und Vorteile geworben. Blickt man zurück auf die Geschichte von Smart-Home-Lösungen bzw. klassischer Heimautomatisierung, handelte es sich dabei vor allem um Lösungen für technik-affine 
Menschen. Zudem war die Automatisierung des eigenen Heims mit enormen Kosten für benötigte Technik und Umbauten verbunden (vgl. Bachfeld \& Hansen, 2013). Mittlerweile werben Anbieter von Smart-Home-Lösungen jedoch mit einer einfachen Installation und Bedienung der Geräte und bieten Lösungen in einem bezahlbaren Preissegment an.

Durch die damit einhergehende Verbreitung gehen jedoch auch diverse Herausforderungen einher, um die Nutzbarkeit der Systeme durch nicht-technik-affine Menschen sicherzustellen. In diesem Paper sollen eben jene Herausforderungen zusammengefasst werden (s. Kapitel 2). Daran anknüpfend wird in Kapitel 3 das Eclipse Smart Home Framework als Open-SourceLösung vorgestellt, auf dessen Basis eine Oberfläche entwickelt wird, die vor allem dem Aspekt einer einfachen Einbindung von Geräten durch weniger technik-affine Personen gerecht werden soll. Die Oberflächen-Entwicklung erfolgt im Rahmen eines nutzerzentrierten Designprozesses, dessen Methoden und Ergebnisse in Kapitel 4 beschrieben sind. Basierend auf den dabei gesammelten Erfahrungen erfolgt in Kapitel 5 die Ergebnisdiskussion.

\section{Herausforderungen im Smart Home Umfeld}

Die Hard- und Softwarelösungen für Smart-Home-Geräte und -Anwendungen sind heutzutage vielfältig. Dies geht einerseits damit einher, dass die bereitgestellten Anwendungen von verschiedenen Herstellern auch unterschiedliche Interaktionskonzepte besitzen. Weiterhin ergibt sich ein Netzwerk, in dem manche Geräte stark, andere wiederum nur schwach zusammenhängend sind (UX Magazine, 2013). Stark zusammenhängend werden die Geräte vor allem durch gemeinsame Nutzungsszenarien. Dies ist beispielsweise der Fall, wenn der Nutzer möchte, dass automatisch das Licht angeht, wenn die Rollläden herunterfahren. Schwach zusammenhängende Geräte werden hingegen unabhängig voneinander genutzt, beispielsweise Heizungen und Waschmaschinen.

Die Vielfältigkeit von sowie auch Abhängigkeit zwischen den verschiedenen Geräten führen zu einer weiteren Herausforderung: Sowohl das physische Gerät, als auch die Anwendung zur Steuerung müssen von den jeweiligen Nutzern bedient werden können. Einheitliche Normen oder Standards an die Benutzerführung existieren diesbezüglich noch nicht, weshalb sich der Benutzer für jedes Gerät neu mit der Steuerung und den zur Verfügung stehenden Interaktionselementen vertraut machen muss (UX Magazine, 2013).

Die weite Verbreitung auf dem Massenmarkt führt zudem dazu, dass die Geräte und Anwendungen für eine äußerst breite Nutzergruppe zugänglich sein müssen: zu den „neuen“ Nutzern zählen nun auch Kleinkinder oder Senioren, entweder als direkte Nutzer oder als indirekte, wenn andere Haushaltsmitglieder entsprechende Geräte installiert haben. Diese unterschiedlichen Nutzergruppen müssen in Bezug auf ihre (technischen) Erfahrungen sowie ihre körperlichen und geistigen Fähigkeiten betrachtet werden (Engelbrecht et al., 2014).

Eine mit dem bereits beschriebenen Zusammenwirken verschiedener Geräte zusammenspielende Herausforderung bildet der Bereich um Nutzungsszenarien und Regeln. Dabei erstellt und steuert der Nutzer automatisierte Abläufe. Ein beispielhaft skizzierter 
Ablauf für einen Morgen könnte wie folgt aussehen: Um 7:00 Uhr dimmt das Licht langsam höher, sodass um 7:15 Uhr die volle Lichtintensität erreicht ist. Gleichzeitig wird um 7:00 Uhr die Heizung im Badezimmer vorgewärmt und um 7:20 Uhr fahren die Rollläden im Schlafzimmer hoch. Bei der Erstellung solcher Systeme muss berücksichtigt werden, dass der Nutzer in einen Nutzungszwang geraten kann, der sein Verhalten beeinflusst. Steht beispielsweise jeden Morgen ein Kaffee bereit, so wird der Nutzer diesen wahrscheinlich auch trinken - weil er da ist, nicht aber, weil der Nutzer Lust auf Kaffee hat. Solche fest eingestellten Abläufe müssen sich zudem flexibel anpassen lassen, falls sich der Nutzungskontext ändert: Ist der Nutzer im Urlaub oder krank, wird er wahrscheinlich nicht wollen, dass alle eingestellten Abläufe weiterhin ausgeführt werden.

Den oben aufgeführten Herausforderungen zu begegnen, ist Ziel der Entwicklung einer auf dem Eclipse Smart Home Framework aufbauenden Benutzeroberfläche, die Nutzern einheitliche Wege für die Installation und Steuerung von Smart-Home-Geräten bietet. Das Projekt wird nachfolgend beschrieben.

\section{Das Projekt}

Das Eclipse Smart Home Projekt ist ein Open-Source Framework für die Hausautomatisierung, dessen Entwicklung von der Telekom getrieben wird. Das Projekt existiert seit Anfang 2014. Die itemis AG ist als Mitglied der „Eclipse Internet of Things Industry Working Group“ aktiv an der Entwicklung des Frameworks beteiligt.

Der Grundgedanke des Projekts besteht darin, die Entwicklung von Smart-Home-Lösungen in einem heterogenen Umfeld zu vereinfachen, wenn verschiedene Protokolle und Standards unterschiedlicher Smart-Home-Geräte kombiniert werden sollen. Dazu wird für jedes Gerät, mit dem kommuniziert werden soll, ein sogenanntes „Binding“ implementiert, das das Gerät mit dem Framework bekannt macht, sodass es sich über eine einheitliche Oberfläche steuern und mit anderen Komponenten vernetzen lässt.

Die Entwicklung einer solchen Oberfläche ist Ziel eines internen Entwicklungsprojektes der itemis AG. Ausgangspunkt für dieses Projekt war die Feststellung eines Mitentwicklers des Eclipse Smart Home Frameworks, dass es keine geeignete Oberfläche für dieses gibt, die weniger technisch affinen Nutzern eine einfache Einbindung von Geräten ermöglicht. Eine erste Version der Oberfläche wurde zunächst vom entsprechenden Entwickler ohne Einbezug von Usability-Methoden umgesetzt und erst zu einem späteren Zeitpunkt dem UsabilityTeam der itemis AG zur näheren Analyse und Verbesserung vorgelegt. Grund für die Zusammenarbeit war die Feststellung des Entwicklers, dass ihm nicht genau bekannt war, was die Nutzer tatsächlich benötigen und wie er diesen die einzelnen Funktionen benutzergerecht zur Verfügung stellen und anzeigen kann.

Die Benutzeroberfläche und die dahinter stehende Anwendung adressieren verschiedene Use Cases. Der Fokus liegt primär auf einer einfachen Anbindung verschiedener Smart-HomeGeräte. Ziel ist es, geräteunabhängige, verständliche und einheitliche Wege anzubieten, die Geräte mit der Anwendung zu verbinden. Darüber hinaus kann über die Benutzeroberfläche 
auf alle konfigurierten Geräte zugegriffen und diese gesteuert werden. In der Oberfläche stehen dem Nutzer darüber hinaus verschiedene Konfigurationsmöglichkeiten zur Verfügung. Dabei geht es insbesondere um Möglichkeiten der Darstellung der angebundenen Geräte, z. B. durch die Organisation dieser in verschiedenen Gruppen. Auch soll die Anwendung zukünftig die Möglichkeit bieten, Regeln bezüglich des Zusammenspiels verschiedener integrierter Smart-Home-Geräte zu erstellen und zu visualisieren.

Durch den laufenden Entwicklungsprozess konnten bereits erste Erkenntnisse hinsichtlich dieser Use Cases gewonnen werden. Diese sind im nächsten Kapitel beschrieben.

\section{Evaluation der Smart Home Anwendung}

Um die Gebrauchstauglichkeit der Eclipse-Smart-Oberfläche sicherzustellen, werden gemäß DIN EN ISO 9241-11 drei grundsätzliche Ziele verfolgt: Erstes Ziel ist, dem Nutzer die Funktionen zur Verfügung zu stellen, die er benötigt, sodass er die von ihm gewünschten Aktionen ausführen kann (Effektivität). Dazu zählen primär das Anschließen und Steuern von Geräten, die er in seinem Smart Home im Einsatz hat oder einsetzen möchte. Darüber hinaus soll er dies in einer angemessenen Zeit schaffen können (Effizienz), weshalb ein möglichst einheitliches, geräteübergreifendes Konzept für Setup und Steuerung angeboten werden sollte. Drittens liegt der Fokus auf der Sicherstellung der Verständlichkeit der Anwendung sowie der Zufriedenheit des Nutzers während des Gebrauchs.

Verschiedene Usability-Methoden sollten dabei helfen, diese Ziele zu erreichen (s. Abb. 1). Da vor dem Start der Einbindung von Usability-Maßnahmen in den Entwicklungsprozess der Benutzeroberfläche bereits eine erste Version vorlag, diente eine Heuristische Evaluation als Einstiegspunkt. Für entsprechende Evaluationen eignen sich die zehn Kriterien zur Bewertung der Usability in Smart-Home-Umgebungen aus Engelbrecht et al. (2014, S. 11). Grundsätzlich konnte durch die heuristische Evaluation festgestellt werden, dass die Oberfläche zwar die Möglichkeit bietet, alle Smart-Home-Geräte, für die ein Binding existiert, mit der Anwendung zu verbinden und diese zu steuern; allerdings auf eine umständliche und für den Nutzer nicht nachvollziehbare Art. Das bis zu diesem Zeitpunkt umgesetzte Interaktionskonzept verlangte vom Nutzer viele unnötigen Interaktionsschritte, bis ein Gerät tatsächlich verbunden und der Nutzer somit in der Lage war, dieses zu steuern. Bereits basierend auf den Ergebnissen der heuristischen Evaluation wurde die Benutzeroberfläche grundlegend überarbeitet. Die aktualisierte Version wurde anschließend in einem Usability-Test mit typischen Endanwendern evaluiert. Da das Feedback der Evaluation aufzeigte, dass insbesondere die Anbindung von Smart-Home-Geräten an die Anwendung für die meisten Probleme sorgt, wurde das Interaktionskonzept für den SetupProzess vollständig überarbeitet und in einem weiteren Usability-Test basierend auf Papierprototypen getestet. Nachfolgend werden die durchgeführten Usability-Tests mit ihren Ergebnissen sowie das resultierte Interaktionskonzept im Detail beschrieben. 


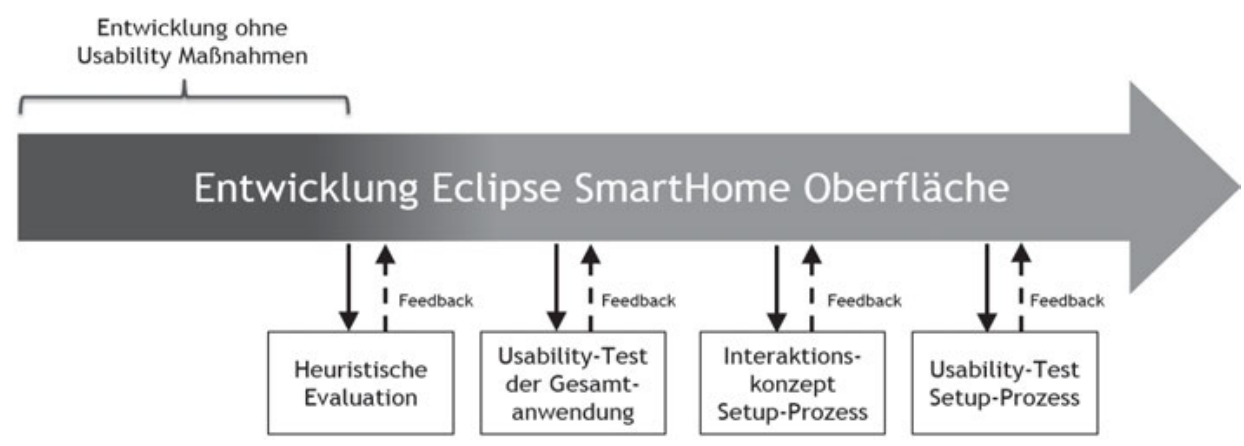

Abbildung 1: Bisherige Aktivitäten im Rahmen des Usability-Engineering-Prozesses

\section{Usability-Test der Gesamtanwendung}

Die Integration echter Nutzer in den Entwicklungsprozess ist zwingend erforderlich - am besten bereits vor Beginn der Entwicklung, um die Nutzer und deren Aufgaben innerhalb des Nutzungskontexts $\mathrm{zu}$ verstehen und basierend auf diesen Erkenntnissen die Benutzerschnittstelle zu entwerfen. Wie in der Praxis leider häufig anzutreffen ist, startete die Entwicklung auch hier ohne jeglichen Einbezug von Nutzern allein basierend auf den Erfahrungen, Kenntnissen und - auch gestalterischen - Fähigkeiten der Entwickler. Bereits die heuristische Evaluation hat gezeigt, dass diese Vorgehensweise massive Nutzungsprobleme mit sich gebracht hat. Ein Usability-Test sollte daher untersuchen, wie die Nutzer grundsätzlich mit der Oberfläche zurechtkommen, aber auch, um dem Entwickler aufzuzeigen, wo große Schwachstellen existieren. Dabei wurde Wert darauf gelegt, Nutzer mit verschiedenem Kenntnisstand zu rekrutieren, um mögliche Einflüsse in die Analyse einbeziehen zu können. Zwei der rekrutierten Probanden wiesen noch keine praktische Erfahrung mit Smart-Home-Anwendungen auf, interessierten sich jedoch stark für das Thema. Die anderen drei Probanden haben bereits Smart-Home-Geräte genutzt, wobei zwei dieser Probanden eigene Geräte in ihrem Haushalt installiert haben.

Im Rahmen des Usability-Tests sollten die Probanden zwei Aufgaben erfüllen: (1) das Entpacken und physische sowie virtuelle Anschließen einer Philipps Hue, einem System bestehend aus einer Bridge und einer Lampe, sowie (2) die Steuerung der Hue Lampe (Lampe einschalten und blau leuchten lassen). Keiner der Probanden hatte zuvor die Philipps Hue verwendet, sodass der Kenntnisstand der Probanden diesbezüglich gleich war.

Die Ergebnisse des Usability-Tests zeigten, dass kein Proband ohne Hilfe in der Lage war, die Philipps Hue anzuschließen und die Lampe zu steuern. Wesentliche Probleme wurden vor allem durch technische Bezeichnungen in der Oberfläche verursacht. So wurde hier eine Unterscheidung in „Things“, „Items“ und „Bindings“ vorgenommen, die für die Nutzer nicht verständlich war. Diese Begriffe betiteln Bereiche in der Oberfläche, in denen Konfigurationen vorgenommen werden müssen, um die Hue anzuschließen. So muss im Bereich der „Bindings“ das Protokoll für die Hue ausgewählt werden. Im Bereich „Items“ musste die Bridge konfiguriert sowie eine Gruppe angelegt werden, der die Lampe später 
zugefügt werden sollte. Erst im Bereich „Things“ konnte dann die konkrete Lampe hinzugefügt werden.

Das bis zu diesem Zeitpunkt vorhandene Konzept wurde von keinem Nutzer verstanden. Dies zeigte vor allem, dass sich die mentalen Modelle vom Entwickler sowie von den Nutzern unterscheiden. Weiterhin wurde deutlich, wie wesentlich eine klare Benutzerführung ist, die dem Nutzer genau verdeutlicht, wie er das Smart-Home-Gerät anschließen muss. Insbesondere bei der Hue wurde dies deutlich, da - anders als bei direkt WLAN-fähigen Geräten - zunächst die Bridge als Empfangsstation (vergleichbar mit einem Router) angeschlossen werden muss und erst dann die tatsächliche Lampe in der Oberfläche erkannt werden kann. Diese Trennung der Geräte führte bei allen Probanden zu Verwirrung. Weiterhin zeigte sich deutlich, dass es beim Thema Smart Home nicht nur um die Usability der Benutzeroberfläche geht, sondern auch um die des verwendeten Smart-Home-Geräts. So hatte es ein Proband zwar mit Hilfestellung geschafft, die Hue in die Benutzeroberfläche zu integrieren, konnte diese aber nicht steuern - der physikalische Schalter der Lampe stand auf „Aus“. Die eigentliche Steuerung der Lampen führte $\mathrm{zu}$ keinen Problemen bei den Probanden. Dies erfolgt in einem separaten Control-Bereich (s. Abb. 2) mittels Schiebereglern und An-/Aus-Schaltern. Angemerkt wurden diesbezüglich vor allem Aspekte der Benennung: so wurden zum Teil gleiche Benennungen für inhaltlich unterschiedliche Aktionen verwendet.

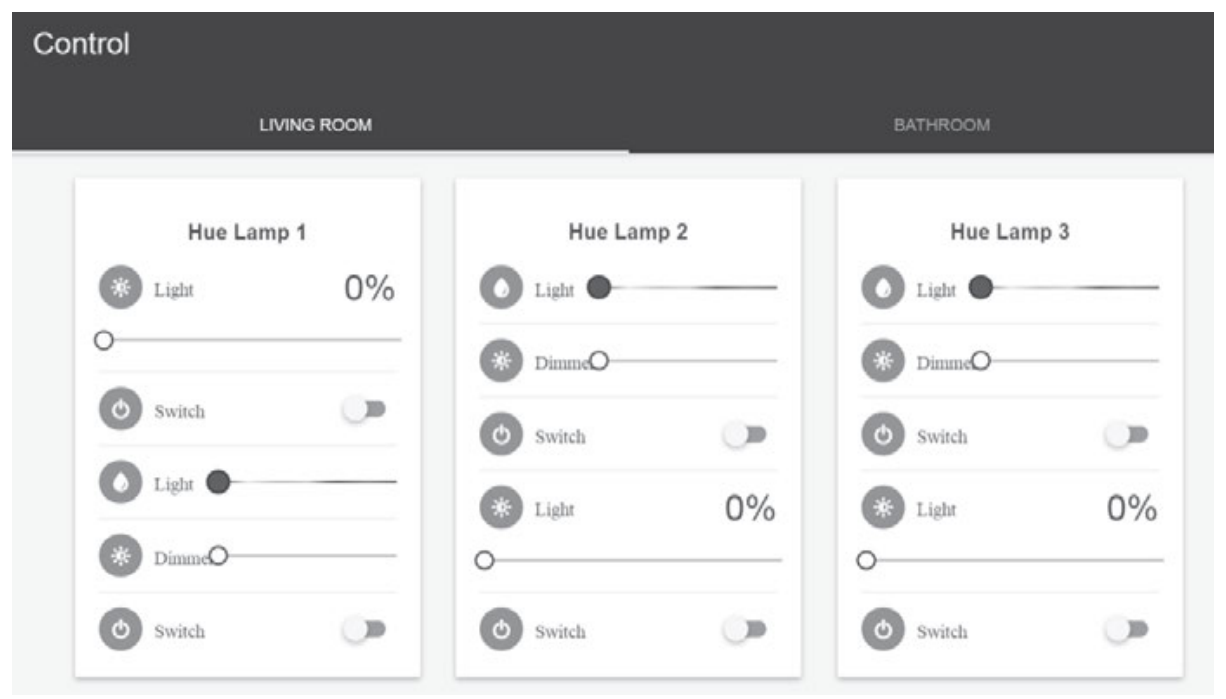

Abbildung 2: Control-Bereich zur Steuerung angeschlossener Geräte

\section{Interaktionskonzept Setup-Prozess}

Da der Setup-Prozess die Nutzer vor die größte Hürde gestellt hat, wurde der Fokus im weiteren Vorgehen auf die Optimierung des Gerätesetups gesetzt. Basierend auf den Erkenntnissen aus dem ersten Usability-Test wurde innerhalb des Usability Engineering 
Teams ein neues Interaktionskonzept für diesen Prozess entwickelt. Neben den Erfahrungen der Teammitglieder wurden darüber hinaus Interaktionskonzepte vergleichbarer Systeme zur Steuerung von Smart-Home-Objekten bei der Gestaltung berücksichtigt. Da in Kapitel 2 als eine wesentliche Herausforderung herausgestellt wurde, dass Interaktionskonzepte zwischen den verschiedenen existierenden Systemen variieren, wurde als wichtig erachtet, in der Benutzeroberfläche ein einheitliches Vorgehen für alle Geräte anzubieten. Dazu wurde zuerst ein Grobkonzept entworfen, das den kürzesten Weg zum Ziel - also die Mindestanzahl an nötigen Schritten - wiederspiegelt, die ein Nutzer durchführen muss, um ein Gerät mit der Anwendung $\mathrm{zu}$ verbinden und in den Kontrollbereich $\mathrm{zu}$ gelangen. Auf Basis dieses Konzepts wurden weitere Handlungsabläufe aufgenommen, um auch vom Idealfall abweichende Situationen darstellen zu können. Dies sind z. B. Situationen, in denen ein Gerät nicht direkt WLAN-fähig ist und eine zusätzliche Empfangsstation benötigt wird, oder ein Gerät nicht automatisch von der Anwendung erkannt wird und somit manuell hinzugefügt werden muss.

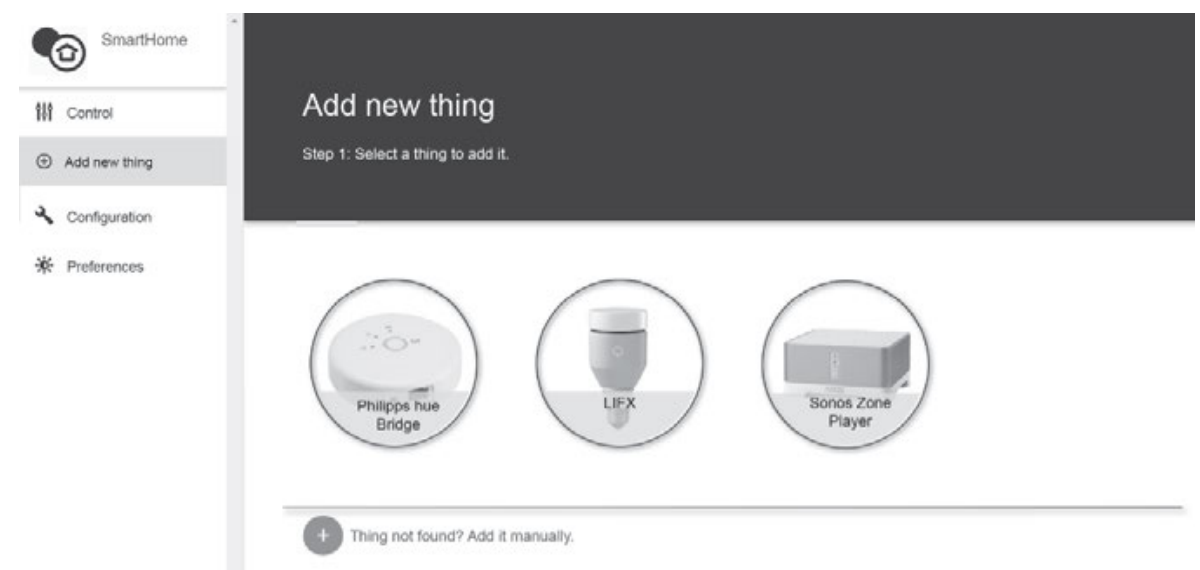

Abbildung 3: Schritt 1 im Wizard zum Gerätesetup

Der neue Setup-Prozess ist als Wizard aufgebaut, der dem Nutzer klar beschreibt, was dieser als nächsten Schritt ausführen muss. Essentiell für die Unterstützung von weniger technikaffinen Nutzern ist, dass die Anwendung Smart-Home-Geräte automatisch erkennt, wenn der Nutzer diese dem Netzwerk hinzufügt, und anschließend im Bereich „Add new thing“ (s. Abb. 3) anzeigt. Darüber hinaus wurde in dem neuen Interaktionskonzept viel visuell gearbeitet. Zum Beispiel werden die einzelnen Smart-Home-Objekte durch Fotos dieser repräsentiert. Diese Abbildungen sollen dem Nutzer dabei helfen, die Geräte zu erkennen und zu verstehen, welches Gerät er in welcher Reihenfolge mit der Oberfläche verbinden muss. So wird ihm z. B. zunächst nur die Hue Bridge angezeigt und erst nach deren Anschließen die einzelnen Lampen. Durch Selektion eines der angezeigten Geräte leitet die Anwendung den Nutzer weiter durch den Prozess. Im nachfolgenden Schritt hat er die Möglichkeit, dem Gerät einen eigenen Namen zu geben. Handelt es sich um Geräte ähnlich einer Empfangsstation wie die Hue Bridge, werden dem Nutzer anschließend alle zur Verfügung stehenden Geräte, welche von der Station erkannt wurden, angezeigt, damit auch 
diese der Anwendung hinzugefügt werden können. Hier kann er auswählen, welches Gerät er anschließen möchte und auch diesem einen eigenen Namen geben. Handelte es sich beim ersten Schritt um ein Gerät, welches direkt bedient werden kann (direkt WLAN-fähig), entfällt dieser Schritt. Zuletzt hat der Nutzer die Möglichkeit, die zugefügten Geräte einer bestehenden Gruppe (z. B. Wohnzimmer) zuzufügen oder zunächst eine neue Gruppe anzulegen. Diese Gruppen sind zentral für den Control-Bereich, da die Geräte dort basierend auf den Gruppen geordnet sind (s. Abb. 2). Abschließend erhält der Nutzer eine Bestätigung, dass das Gerät hinzugefügt wurde und wird zum Control-Bereich geleitet, in dem er dann mit dem Gerät interagieren kann.

Falls die Anwendung ein Smart-Home-Gerät nicht automatisch erkennt, kann der Nutzer dieses manuell hinzufügen. Dazu kann er aus allen in der Oberfläche verfügbaren Bindings das auswählen, für das er ein Gerät anschließen möchte. Auch hier wird wieder Gebrauch von Abbildungen gemacht, um den Nutzer visuell bei der Auswahl zu unterstützen und nicht durch technische Begriffe zu verwirren. Nachfolgend wählt er wiederum entweder ein konkretes Gerät aus, wenn dieses direkt WLAN-fähig ist, oder zunächst eine Empfangsstation. Der einzige Unterschied zur automatischen Erkennung ist hier, dass der Nutzer je nach Binding manuelle Eingaben machen muss, etwa die Eingabe einer IP-Adresse oder Geräte-ID.

\section{Usability-Test des Setup-Prozesses}

Im Rahmen einer zweiten Usability-Evaluation wurde das neue Interaktionskonzept für den Setup-Prozess überprüft. Das Konzept selbst wurde zunächst in Form eines Papierprototypen umgesetzt, um frühes Nutzerfeedback noch vor der Implementierung zu erhalten. Für diesen Usability-Test wurden weitere fünf Endnutzer rekrutiert, die hinsichtlich der Vorerfahrung mit Smart Home der Nutzergruppe aus dem ersten Test gleichen. Die Probanden sollten einerseits eine Hue (Bridge und Lampe) und andererseits eine LIFX (Lampe ohne Bridge) anschließen. Grundsätzlich erkennt die Anwendung beide Lampen automatisch, für den Test wurde allerdings simuliert, dass die LIFX nicht erkannt und somit manuell durch die Eingabe der Device-ID angeschlossen werden muss. Die System Usability Scale (Brooke, 1996) sollte anschließend einen Eindruck über die subjektive Einschätzung der Usability und Learnability der Oberfläche durch die Probanden liefern.

Grundsätzlich konnte durch den Usability-Test gezeigt werden, dass der neue Setup-Prozess den Nutzer deutlich besser anleitet, als dies zuvor der Fall war. Die Hue konnte von allen Probanden schnell und problemlos angeschlossen werden. Dabei unterstützten laut der Nutzer vor allem die Abbildungen der Bridge und der Lampen sowie die klare Benutzerführung, was in welcher Reihenfolge angeschlossen werden muss. Diese Benutzerführung half den Nutzern auch bei der manuellen Installation der LIFX. Interessant war hier, dass der Begriff des „Bindings“ keine Probleme verursachte, da sich die Probanden an den Abbildungen der Geräte orientierten und für sie dadurch klar war, was sie auswählen sollten. Problematisch war vielmehr, die Device-ID einzugeben, da die Probanden hier nicht wussten, an welcher Stelle diese auf der Verpackung des Geräts zu finden ist. Grundsätzlich konnte durch den neuen Setup-Prozess ein SUS-Score von 76,5 (SD = 9.1) erreicht werden, wobei die Usability mit 72,5 und die Learnability mit 92,5 bewertet wurde. 


\section{Diskussion und Implikationen}

Die neu entwickelte Benutzeroberfläche soll verschiedenen Herausforderungen, die mit Smart Home einhergehen (s. Kapitel 2), entgegen wirken. Aktuell steht der Entwicklungsprozess noch relativ am Anfang. So wurde nach einer Gesamtevaluation der Anwendung zunächst ein Fokus auf einen geräteübergreifenden, einheitlichen Setup-Prozess gelegt, da das Gerätesetup die Probanden vor die größte Hürde stellte. Während viele Hersteller für ihre Geräte eigene Apps zur Verfügung stellen, erlaubt es die Anwendung dagegen, die einzelnen Geräte über eine einzige Oberfläche anzusprechen, was als wesentlicher Vorteil zu sehen ist. Als zentral hat sich für die Gestaltung der Anwendung auch gezeigt, dass Abbildungen einzelner Geräte in der Anwendung für ein Verständnis des Prozesses durch Nutzer, die bisher noch nicht mit Smart Home in Kontakt gekommen sind, unterstützend wirken. Dies ist insbesondere hilfreich, wenn vor der Verbindung des eigentlich zu steuernden Geräts Empfangsstationen eingerichtet werden müssen. Eine klare Benutzerführung ist hier essentiell.

Grundsätzlich ist geplant, die Anwendung kontinuierlich weiterzuentwickeln und dabei eine enge Zusammenarbeit zwischen den Entwicklern und dem Usability Engineering Team zu fördern. Wir haben diesbezüglich die Erfahrung gemacht, dass der Mehrwert unserer Usability-Aktivitäten für die Entwickler vor allem durch die Durchführung der UsabilityTests ersichtlich wurde. Zwar erhielten wir schon nach der heuristischen Evaluation das Feedback, dass wir wichtige Aspekte identifiziert hätten, die vorher aus Entwicklersicht nicht aufgefallen seien; die Sichtung realer Nutzerproblemen in den Usability Tests verstärke jedoch die Motivation der Entwickler, weiterhin einen nutzerzentrierten Designprozess zu verfolgen.

Deshalb ist im nächsten Schritt eine Nutzungskontextanalyse angestrebt. Wie bereits erwähnt, wurde diese aufgrund des späten Einbezugs des Usability Teams in den Prozess bisher noch nicht durchgeführt. Dies ist nach DIN EN ISO 9241 jedoch zwingend notwendig, um den Nutzer in seinem Kontext sowie seine mentalen Modelle verstehen und darauf basierend Anforderungen ableiten zu können. Neben einer übergreifenden Betrachtung wird hier ein Fokus auf der Regelerstellung und -bearbeitung in der Anwendung liegen, um Ideen zur Begegnung der entsprechend in Kapitel 2 aufgeführten Herausforderungen generieren zu können.

Insgesamt hat sich bereits durch die ersten Evaluationen gezeigt, dass die nutzergerechte Gestaltung von Smart-Home-Anwendungen ein komplexes Feld ist, da verschiedene Faktoren und Herausforderungen berücksichtigt werden müssen. Das Nutzerfeedback zur entwickelten Benutzeroberfläche zeigte jedoch, dass wir bezüglich unserer Arbeit auf dem richtigen Weg sind, eine Anwendung $\mathrm{zu}$ gestalten, die vielen der Herausforderungen begegnet. 


\section{Literatur}

Bachfeld, D. \& Hansen, S. (2013). Home, smart Home! Bequeme Steck- und Funktionslösungen im Einsatz. heise online, 9. http://www.heise.de/ct/artikel/Home-smart-Home-1834136.html (Stand: 29.05.2015).

Brooke, J. (1996). SUS: a ‘quick and dirty' usability scale. In: Jordan, P., Thomas, B., Weerdmeester, B., McClelland, I. (Hrsg.), Usability Evaluation in Industry. Taylor \& Francis London, S. 189-194.

Engelbrecht, K.-P., Ehrenbrink, P., Hillmann, S. \& Möller, S. (2014). Messung und Bewertung der Usability in Smart Home-Umgebungen, In VDE Kongress 2014. Smart Cities - Intelligente Lösungen für das Leben in der Zukunft. Frankfurt am Main.

Statista (2015). Installed base of home automation/smart home systems in Europe from 2012 to 2019 (in millions). http://www.statista.com/statistics/286815/smart-home-systems-installed-in-europe/ (Stand: 29.05.2015)

UX Magazine (2013). Home Automation: The next frontier for UX? UX Magazine, 966, http://uxmag.com/articles/home-automation-the-next-frontier-for-ux (Stand: 29.05.2015). 